\title{
LAS FUNCIONES EVIDENCIALES DEL MARCADOR PRAGMÁTICO COMO (QUE) EN EL HABLA ORAL DEL ESPAÑOL DE CHILE ${ }^{1}$
}

\author{
Geraldine QUARTARARO \\ Pontificia Universidad Católica de Chile \\ Carlos GonZÁlez Vergara \\ Pontificia Universidad Católica de Chile
}

\section{RESUMEN}

Este artículo analiza las funciones del marcador pragmático como (que) en el español de Chile. El análisis muestra tanto las funciones de como (que) ya señaladas en estudios previos (Acín Villa 1987; Jørgensen 2012; Puga 1997) como algunas funciones evidenciales indirectas que no habían sido detectadas previamente. En este sentido, como (que) puede funcionar como un marcador evidencial de inferencia, un marcador evidencial de suposición y un marcador evidencial referido de segunda mano. El análisis cuantitativo de como (que) muestra además: (1) que el marcador, independientemente de su significado, se utiliza con mayor frecuencia entre los hablantes jóvenes; (2) que en la mayoría de los casos es más frecuente en el discurso de las mujeres, y, finalmente, (3) que su uso disminuye proporcionalmente al aumento en la edad de los participantes. Los datos de primera mano usados para este análisis fueron elicitados a través de la tarea Family Problems Picture (San Roque et al. 2012). Todas las transcripciones de los datos han sido recopiladas en un nuevo corpus de la variedad del español chileno.

PALABRAS ClaVE: español de Chile, marcador pragmático, evidencialidad indirecta.

\section{AbSTRACT}

This paper explores the functions of the pragmatic marker como (que) in Chilean Spanish. The analysis displays both the functions of como (que) already described in previous studies (Acín Villa 1987; Jørgensen 2012; Puga 1997) and some previously unnoticed indirect evidential functions. In this sense, como (que) can operate as an inferred evidential marker, an assumed evidential marker, and

${ }^{1}$ Estudio inserto en el Proyecto Conicyt-Fondecyt de Posdoctorado n. ${ }^{\circ} 3180009$ «La evidencialidad en el español de Chile en contacto con el aimara».

ORALIA, vol. 23/2, 2020, págs. 319-340. 
a second-hand reported evidential marker. Furthermore, the quantitative analysis presented here shows that (1) como (que), regardless of its meaning, is expressed more frequently among young speakers; (2) it is almost in all cases more frequent in women's speech; and, finally, (3) its use decreases proportionally to the increase in the age of the participants. The original data used for this analysis were gathered through the Family Problems Picture task (San Roque et al. 2012). All the data transcripts were used to compile a novel corpus of Chilean Spanish.

KEYWORDS: Chilean Spanish, pragmatic marker, indirect evidentiality.

Fecha de recepción: 05/06/2019

Fecha de aceptación: 22/10/2019

Fecha de la versión definitiva: 05/11/2019

\section{INTRODUCCIÓN ${ }^{2}$}

La partícula como es el resultado de la contracción del adverbio de modo latino quōmodo. En la bibliografía (Acín Villa 1987; Iglesias Bango 2005; Rodríguez 2009) hay un acuerdo generalizado en cuanto a sus funciones gramaticales: como puede tener función de adverbio, conjunción y preposición. Cuando funciona como adverbio, al igual que su antecedente latino, como es un adverbio relativo de modo y aparece junto a un antecedente tanto explícito como implícito (1). Cuando tiene valor de conjunción, como aparece en oraciones adverbiales (causales y condicionales) (2). Finalmente, con valor de preposición, como precede a partes del discurso con respecto a las cuales señala cierta forma de adscripción o semejanza (3).

(1) No le gustó la manera como se lo dijo (Hernando Cuadrado 2002: 326).

(2) Como se le estropeó el coche, llegó tarde a la oficina (Hernando Cuadrado 2002: 332).

(3) Ingresó como alumno en la Academia Militar (Hernando Cuadrado 2002: $336)$.

A estas funciones gramaticales, es necesario añadir una serie de funciones semántico-pragmáticas derivadas. Como ha sido descrito como un recurso léxico de atenuación (Montes 1980; Puga 1997; Cervera Rodríguez 2009); sin embargo, estudios más recientes han mostrado que se trata de un

${ }^{2}$ Este artículo es el resultado del trabajo conjunto de los dos autores. La recolección de datos y su transcripción fue realizada por Geraldine Quartararo, quien se ocupó de la redacción de los apartados 1, 2, 2.1 y 2.2. Carlos González Vergara se ocupó de la redacción del apartado 2.3. Los apartados 0 y 3, así como el análisis, fueron redactados conjuntamente por los dos autores. 
marcador pragmático plurifuncional cuyo valor depende estrictamente del contexto sintáctico y comunicativo en el que se utiliza. Jørgensen (2012) identifica varias funciones discursivas de como: reformulación, explicación, retardación, aproximación, ejemplificación, atenuación, intensificación y citación. A esta amplia gama de funciones semántico-pragmáticas, este análisis añade otra: la función evidencial indirecta. Nuestros datos ${ }^{3}$ muestran que en muchas instancias como se utiliza para introducir una información con la que el hablante ha tenido un contacto indirecto. Este contacto indirecto puede indicar tanto una inferencia/suposición hecha por parte del hablante con respecto a la situación que está describiendo (4) como ser el resultado de un reporte (5).

(4) P1: Ah / mmm (4") cinco personaas // comooo parecen ser / amigos // yyy como de la misma edad // de / mmm entre veinte y treinta / por ahí / eee // tomandooo / a mí parece / ¿cerveza? / Y conversando $[\ldots]$

(5) P3: [...] yyy después de que lo llevan preso / eee / ya en la cárcel como que reflexiona de su actitud / y de la vida / y suh acciones / y después de salir / vuelve a familia pero la familia ya no lo acepta / y esa es la historia ${ }^{4}$

Esta hipótesis, relativa a la función evidencial de como, está corroborada por el análisis comparativo de like y como de Jørgensen y Stenström (2009), quienes señalan una correspondencia funcional entre las dos partículas en español y en inglés, y por el análisis del marcador discursivo like propuesta por Andersen: «... it encodes a procedure in the sense of a way of guiding, or constraining, the inferential phase of comprehension» (2000: 18).

Usos pragmáticos del marcador como (que) están documentados en las variedades del español americano habladas en Perú, Ecuador, Venezuela, Guatemala, México y República Dominicana (Kany 1945: 383) y, además, en el español madrileño (Jørgensen 2012), en el español de Colombia (Montes 1980) y en el español de Chile (Jørgensen 2012; Puga 1997, 2014; Panussis Lyon y San Martín Núñez 2017).

A partir de las descripciones existentes sobre el tema en las variedades del español en general y en la variedad del español de Chile en particular ${ }^{5}$, los objetivos principales que este estudio pretende alcanzar son:

${ }^{3}$ Los materiales analizados en el presente estudio provienen de un corpus que ha sido recolectado en la ciudad de Santiago de Chile en 2018. Se trata de materiales no publicados anteriormente que se presentan aquí por primera vez. Para más información véase la subsección 2.1.

${ }^{4}$ El ejemplo (5) será presentado y mostrado como ejemplo (17) en el apartado 2.2.

5 Tal como se verá en detalle en la sección 2.3, el marcador como (que) es especialmente interesante de analizar en la variedad chilena de español, dada su alta frecuencia relativa. 
i) establecer las funciones semántico-pragmáticas de como en el habla oral chilena y

ii) delinear la relación entre las características sociolingüísticas de los hablantes y las funciones semántico-pragmáticas de como.

Para alcanzar estos dos objetivos analizaremos un corpus oral de la variedad del español de Chile recopilado en Santiago de Chile en 2018 y elicitado a través de la tarea Family Problems Picture. Las cuestiones que nos proponemos responder son las siguientes:

1) ¿Qué funciones pragmáticas de como (que) pueden encontrarse en nuestro corpus del español de Chile?

2) ¿Qué funciones se dan con mayor frecuencia? ¿Son frecuentes las funciones evidenciales?

3) ¿Existe una relación entre las funciones de como (que) y las características sociolingüísticas de los hablantes?

4) ¿Existe una relación entre las funciones de como (que) y las características de la tarea propuesta? ${ }^{6}$

Este trabajo está estructurado de la siguiente manera: la introducción delimita el tema y presenta los objetivos del estudio; la sección 1 proporciona una descripción general de los marcadores pragmáticos, del dominio evidencial y de su relación con el modal. La sección 2 presenta el análisis de como y muestra los resultados del análisis. En particular, la subsección 2.1 describe el proceso de recopilación y las características del corpus, la subsección 2.2 delinea y ejemplifica las funciones de como detectadas en el corpus, contestando a las preguntas (1) y (2), y la subsección 2.3 muestra el análisis cuantitativo y contesta a las preguntas (3) y (4). Finalmente, la sección 3 proporciona algunas consideraciones conclusivas sobre los resultados.

A partir de aquí nos referiremos a la construcción analizada a través de la indicación como (que), ya que en los datos la partícula como se presenta como una variante formal dependiente de factores sintácticos y no funcionales de la construcción como (que).

(6) P2: [...] como que no están de acuerdo que él llegue / tan contento a saludar $[\ldots]$

(7) P1: aa la ropa se la devuelve / aaa tienes razón / además tiene una sonrisa como contento / entre todas las cosas [que le pasaron]

\footnotetext{
${ }^{6}$ Dado que, como se señala en 2.1, la tarea Family Problems Picture está diseñada para elicitar diversos comportamientos lingüísticos relacionados con la expresión de la fuente de información, este objetivo busca describir si, en efecto, como (que) codifica diferentes informaciones evidenciales de acuerdo con cada tarea exigida.
} 


\section{MARCADORES PRAGMÁTICOS, EVIDENCIALIDAD Y MODALIDAD}

Los marcadores pragmáticos han sido ampliamente estudiados y su definición no es unánime. Desarrollaremos este análisis en el marco de la clasificación de Fraser (1996, 2006) que es la que mejor da cuenta de las funciones semántico-pragmáticas desempeñadas por como (que) en nuestros datos, ya que identifica por separado la mayoría de las funciones de como (que) identificadas en el análisis ${ }^{7}$. Fraser (2006) define los marcadores pragmáticos como partes del discurso que no forman parte del contenido proposicional del mensaje transmitido y no contribuyen al significado de la proposición per se. El autor identifica cuatro tipos de marcadores:

i) los 'marcadores pragmáticos básicos', que señalan la fuerza ilocutiva del enunciado;

ii) los 'marcadores pragmáticos de comentario', que proporcionan un comentario sobre el mensaje, o una parte del mismo;

iii) los 'marcadores paralelos', que añaden un nuevo mensaje al primero; finalmente,

iv) los 'marcadores del discurso', que especifican la relación entre dos mensajes.

En este estudio, nos centraremos en los marcadores pragmáticos de comentario y en los marcadores del discurso ya que estos dos tipos de marcadores representan las funciones semántico-pragmáticas que como (que) con mayor frecuencia desempeña en los datos.

$\mathrm{Al}$ tener en cuenta la plurifuncionalidad de los marcadores pragmáticos en general y los usos evidenciales de como (que) en particular, utilizaremos además la clasificación de los evidenciales de Aikhenvald (2004). A través del término evidencialidad, la autora define la expresión de la fuente de información, es decir, la expresión de la manera en la que el hablante ha entrado en contacto con la información que está proporcionando. Según Aikhenvald (2004: 65), la evidencialidad se divide en seis subdominios: visual, sensorial, inferencia, suposición, rumores y citación. Estos 6 subdominios pertenecen a dos categorías evidenciales dicotómicas: información directa (subdominio visual y sensorial) e información indirecta (subdominio inferencial, de suposición, de rumor y de citación). Considerando los usos evidenciales de como (que) identificados en este estudio, nos enfocaremos en las características de los subdominios indirectos: inferencial, de suposición y de rumor. A través del término 'inferencial', haremos referencia a cualquier deducción hecha a partir de una evidencia observable, mientras

\footnotetext{
7 Para una aproximación general a la descripción de los marcadores pragmáticos véase Brinton (1996) y Aijmer y Simon-Vandenbergen (2006).
} 
que a través del término 'suposición' nos referiremos a todas las deducciones hechas a partir de un razonamiento basado en algo que el hablante ya sabe. Es relevante especificar que la diferencia entre estos dos subdominios no siempre es realmente clara. Para distinguirlos, consideraremos la siguiente afirmación:

The difference between the 'assumed' evidential $[\ldots]$ and the 'inferred' [...] lies in access to visual evidence of something happening and to the degree of 'reasoning' involved. The less obvious the evidence and the more the speaker has to rely on reasoning based on knowledge or on common sense, the more chance there is that the assumed evidential will be used. An inferred evidential refers to something based on obvious evidence which can be easily observed (Aikhenvald 2004: 2-3).

En cuanto a la discusión de la función evidencial de rumor, considerando los usos de como (que) presentes en el corpus, limitaremos este análisis a la información que se ha alcanzado a través de otra persona de quien el hablante ha escuchado directamente las palabras.

El análisis de los evidenciales no puede prescindir de la evaluación de la relación que este dominio puede tener con el dominio modal epistémico (Chafe 1986: 262; Aikhenvald 2004: 176). Chafe (1986) muestra una visión inclusiva del concepto de evidencialidad definiéndolo, a la vez, como la expresión de la fuente de información (evidencialidad) y como el grado de validación del hablante hacia la información que está proporcionando (modalidad epistémica). A diferencia de Chafe (1986), Aikhenvald (2004) limita su definición de evidencialidad solo a la expresión de la fuente de la información. Es oportuno especificar que los marcadores evidenciales y los marcadores modales expresan, en efecto, dos tipos de indicación sobre la información diferentes, pero conceptualmente cercanos: los evidenciales informan sobre la manera en la que se ha entrado en contacto con la información, mientras que los modales epistémicos indican las evaluaciones del hablante con respecto a la veracidad de la información misma. En las lenguas romances esta cercanía conceptual se manifiesta a través del solapamiento de los dos dominios ${ }^{8}$ que aquí pueden expresarse a través de los mismos morfemas o lexemas (Plungian 2001: 354).

(8) Italiano

[Suonano alla porta] sarà il postino

«[Están llamando a la puerta] será el cartero» (Squartini 2008: 923)

Este solapamiento se manifiesta claramente en los evidenciales indirectos, en estos casos el hablante puede expresar diferentes grados de confia(2001)

${ }^{8}$ Para una aproximación general a la descripción de modalidad epistémica véase Nuyts 
bilidad con respecto a la veracidad de la información debido al tipo de contacto que tuvo con la fuente. Consideramos estas últimas aclaraciones necesarias para los fines del presente estudio dado que en él los autores tomarán una visión inclusiva del concepto de evidencialidad.

\section{El ANÁlisis DE COMO (QUE)}

Las tres subsecciones siguientes describen el análisis de los usos de como (que). La primera (2.1) se centra en la descripción de los materiales. La segunda (2.2) atañe a las funciones semántico-pragmáticas de como (que) dentro del corpus. Finalmente, la tercera (2.3) proporciona un análisis cuantitativo de las funciones de como (que) en relación con las características sociolingüísticas de los hablantes y con las características de la tarea Family Problems Picture.

\subsection{Recopilación de datos}

Los materiales analizados en el presente estudio provienen de un corpus que ha sido recopilado en la ciudad de Santiago de Chile en 2018. Todos los participantes en las sesiones de grabación declararon ser monolingües de español. Los datos que presentamos se elicitaron con el objetivo de estimular la producción de las formas evidenciales. Para este fin, se utilizó la tarea semiestructurada Family Problems Picture (San Roque et al. 2012), que fue diseñada para estimular la evidencialidad y el reporte de habla, pensamientos o emociones (San Roque et al. 2012: 140). La tarea consta de 16 imágenes en blanco y negro, que representan una historia con un orden definido. Sin embargo, la secuencia temporal de la historia no está siempre clara y esto, durante el desarrollo de la tarea, determina procesos de razonamiento e interacciones por parte de los participantes con el fin de entender el orden en el que posicionar las imágenes para construir la historia. La tarea fue desarrollada en cinco pasos. En el primer paso, se pidió a los hablantes que describieran cinco de las 16 imágenes elegidas por nosotros al azar. El contacto directo y visual con las imágenes favorece la expresión de evidenciales directos e inferenciales, si estos existen en la lengua de referencia. En el segundo paso, se solicitó a los hablantes que construyeran una historia con todas las imágenes y les dieran un orden. El propósito de esta parte de la tarea es la activación de procesos de inferencia y suposición. En el tercer paso, se pidió a uno de los dos hablantes que participaron en los dos primeros pasos que describiera la historia construida en primera persona. El cuarto y el quinto paso están directamente relacionados. En el cuarto paso encargamos a uno de los dos hablantes que participaron en 
los tres primeros pasos que relatara la historia construida a un tercer hablante que no había participado en la construcción de la trama. Finalmente, en el quinto paso pedimos al tercer hablante relatar al trabajador de campo la historia que le habían contado. En esta última fase los procesos cognitivos activados permiten el uso de evidenciales referidos.

A través de las transcripciones de las grabaciones se recopiló un corpus de 28953 palabras. El método de transcripción utilizado sigue las convenciones establecidas por el Grupo Val.Es.Co. ${ }^{9}$, especializado en el análisis del español oral. Las transcripciones de las grabaciones fueron realizadas en su totalidad por el mismo trabajador de campo. Al proceso de transcripción siguió un proceso de revisión que fue realizado por nosotros mismos.

En las grabaciones de la variedad del español chileno participaron 30 hablantes monolingües. Este número incluye 16 hombres y 14 mujeres. La edad de los participantes está comprendida entre los 18 y los 92 años. Por razones de análisis, los treinta hablantes fueron agrupados en tres grupos de acuerdo con sus edades: jóvenes (18-30; 12 participantes); adultos (31-50; 14 participantes); y mayores (51 años y más; 4 participantes). La mayoría de los participantes tiene educación superior (29) y solo uno tiene educación media.

\subsection{Las funciones semántico-pragmáticas de como (que)}

La plurifuncionalidad del marcador como (que) sugiere la presencia de un proceso de gramaticalización (Hopper y Traugott 2003) que ha determinado un gradual alejamiento semántico del marcador de su función original de adverbio relativo de modo. Durante este proceso, pese a haber conservado parcialmente su valor comparativo original, como (que) adquirió usos y funciones distintos de los originales. En cuanto a la función evidencial de como (que) detectada en nuestros datos, consideramos que este proceso de reanálisis semántico está constituido por dos partes:

1) el acercamiento comparativo entre la información conocida/percibida/escuchada por el hablante y los pensamientos/conocimientos del mismo hablante con respecto a lo que cree que está dibujado realmente en las imágenes de la tarea, y

2) la evaluación personal del hablante con respecto a la verosimilitud de la información resultante de este proceso comparativo.

${ }^{9}$ El Sistema de transcripción Val.Es.Co. se encuentra disponible en línea en el sitio web $<$ http://www.uv.es/corpusvalesco/convenciones.html>. 
En todos los casos en los que tiene una función evidencial en los datos, como (que) indica la voluntad del hablante de no comprometerse completamente con la veracidad de la información proporcionada. Una explicación similar de como, aunque conceptualmente distinta, se observa en Jørgensen (2012: 218): «como es un marcador discursivo que [...] indica parecido no idéntico con lo dicho, permite al hablante decir algo sin comprometerse a una interpretación literal del enunciado». Consideramos que la disconformidad entre la función de marcador discursivo o de atenuación y la función evidencial indirecta de como (que) reside básicamente en una diferencia en el proceso de acercamiento comparativo mencionado anteriormente, ya que, al trabajar como marcador del discurso, como (que) no señala un acercamiento comparativo entre la información conocida/percibida/escuchada y los pensamientos/conocimiento del hablante, sino que indica un acercamiento comparativo entre la realidad conocida/percibida/escuchada por el hablante y su repertorio lingüístico.

A lo largo de este apartado brindaremos un análisis de las funciones desempeñadas por como (que) en nuestros datos. El Cuadro 1 presenta las funciones a discutir en términos cuantitativos.

CuAdro 1. Funciones de como (que) en los datos

\begin{tabular}{|c|c|c|c|c|c|}
\hline \multicolumn{2}{|c|}{ Funciones de como (que) } & \multicolumn{2}{|c|}{$\mathbf{N}^{\circ}$ Casos } & \multicolumn{2}{|c|}{$\%$} \\
\hline \multicolumn{2}{|c|}{$\begin{array}{l}\text { Funciones gramaticales } \\
\text { (adverbio, conjunción y preposición) }\end{array}$} & \multicolumn{2}{|c|}{68} & \multicolumn{2}{|c|}{10,4} \\
\hline \multicolumn{2}{|c|}{ Función atenuadora } & \multicolumn{2}{|c|}{64} & \multicolumn{2}{|c|}{9,7} \\
\hline \multicolumn{2}{|c|}{ Función ejemplificadora } & \multicolumn{2}{|c|}{4} & \multicolumn{2}{|c|}{0,6} \\
\hline \multicolumn{2}{|c|}{ Función aproximadora } & \multicolumn{2}{|c|}{88} & \multicolumn{2}{|c|}{13,4} \\
\hline \multicolumn{2}{|c|}{ Función retardataria } & \multicolumn{2}{|c|}{15} & \multicolumn{2}{|c|}{2,3} \\
\hline \multirow{4}{*}{$\begin{array}{l}\text { Función } \\
\text { evidencial } \\
\text { indirecta }\end{array}$} & Inferencial & \multirow{4}{*}{380} & 90 & \multirow{4}{*}{58,1} & 13,8 \\
\hline & Suposición & & 255 & & 39,1 \\
\hline & $\begin{array}{l}\text { Ambiguos } \\
\text { (Inferencial/suposición) }\end{array}$ & & 22 & & 3,3 \\
\hline & Rumor & & 13 & & 1,9 \\
\hline \multicolumn{2}{|l|}{ Otros $^{10}$} & \multicolumn{2}{|c|}{37} & \multicolumn{2}{|c|}{5,5} \\
\hline \multicolumn{2}{|l|}{ Total } & \multicolumn{2}{|c|}{652} & \multicolumn{2}{|c|}{100} \\
\hline
\end{tabular}

${ }^{10}$ En este grupo caben todos los casos cuya función no se ha podido identificar debido a interrupciones en la grabación o transcripciones dudosas. 
Con respecto a las funciones semántico-pragmáticas de como (que) en el español de Santiago de Chile, Panussis Lyon y San Martín Núñez (2017) identifican 4 funciones discursivas principales: aproximador, atenuador, rearticulación de relleno e introductor de cita en discurso directo.

Cuando como (que) cumple la función discursiva aproximadora, indica una aproximación terminológica de la cual el hablante no está seguro. En otras palabras, como (que) funciona como un marcador pragmático de comentario de manner-of-speaking (Fraser 1996); esto es, comenta un elemento específico de la oración que el hablante no considera adecuado para la descripción de lo que quiere decir.

(9) P1: = Y pareciera quee la persona estuvo - el hombre adulto estuvo harto tiempo fuera porque trae como un'especie de mochila con su pertenencia / lo que implica que - o que estuvo mucho tiempo fuera

En (9), el uso de como sirve para indicar que el término mochila es, en efecto, una aproximación terminológica. El hablante es consciente que el personaje no está llevando una verdadera mochila; sin embargo, no recuerda la palabra más adecuada para la descripción del objeto, es decir, morral, y llena este vacío terminológico utilizando el término que más se acerca visual y conceptualmente al término adecuado. Esta interpretación de la forma está confirmada además por la presencia de la construcción «una especie de», cuyo uso especifica de manera redundante la incertidumbre del hablante con respecto a la adecuación del término utilizado.

En su función atenuadora, como (que) modera la fuerza ilocutiva del enunciado o mitiga el significado de las palabras sobre las que tiene alcance semántico (Channell 2000; Cutting 2007). Utilizando la terminología de Fraser (1996), en estos casos, como (que) cumple la función de marcador pragmático de comentario de mitigation.

(10) P1: Luego se lo / llevan // los policíah // y ella queda (3") herida / con su hijo en brazo chiquitito ${ }^{\circ}$ (aquí se me imagina que falta algo) ${ }^{\circ}$ está muy brusca la / no sé $(13 ”){ }^{\circ}$ (ahí bueno [entra a la cárcel] $)^{\circ}$

P2: [Esa - esa es como la secuencia]

P1: Sí

En el ejemplo (10), el uso de como disminuye la fuerza ilocutiva del enunciado «esa es la secuencia». A través del término secuencia, P2 indica el orden final de las 16 imágenes de la tarea Family Problems Picture. El uso de como, por lo tanto, resulta necesario para mitigar la fuerza asertiva del enunciado que, en cambio, sin la presencia del marcador, comprometería la imagen social de P2 ya que implicaría que, según él, el orden establecido es el último viable sin otra posibilidad de variaciones por parte de P1. 
Jørgensen (2012) considera la función ejemplificadora de como una subfunción de la función atenuadora que hemos descrito previamente. En contraste con esta postura teórica, Fraser (1996) califica estas dos funciones como distintas e inserta los ejemplificadores dentro del grupo de los marcadores del discurso elaborativos de ejemplificación. En línea con Fraser, en este estudio diferenciaremos estas dos funciones ya que consideramos que operan sobre niveles lingüísticos distintos. Los datos proporcionan un número muy limitado de casos en los que como (que) cumple la función ejemplificadora. Aquí, el marcador introduce una aclaración en referencia a algún elemento que el hablante considera vago o no suficientemente claro (11).

(11) P1: [...] o quizá / tiene que ver con algún tipo de país en particular no sé estoy pensando como // Tailandia o algún país tropical [...]

La función de rearticulación de relleno o retardataria de como (que) está relacionada con un silencio o una pausa larga ${ }^{11}$. En el corpus, cuando como (que) desempeña esta función señala una vacilación en el habla o una pausa del hablante para planificar el discurso (12) ${ }^{12}$.

(12) P2: Es comoo // sí es cuando ya salió / parece que tiene la misma ropa / siempre tiene la misma ropa / no no siempre

Además de las funciones ya mencionadas, este estudio propone una función nueva de como (que): la función evidencial indirecta. Cuando el marcador cumple esta función normalmente puede desempeñar función evidencial de rumor, función evidencial inferencial y función evidencial de suposición. En los términos de Fraser (1996), en estos casos, como (que) funciona como un marcador pragmático de comentario de tipo evidencial -cuando desempeña función evidencial de inferencia y de suposicióny un marcador pragmático de comentario de rumor -cuando desempeña función evidencial de rumor-. Un 3,3\% de casos en los que como (que) funciona como un marcador pragmático de comentario de tipo evidencial no se han podido clasificar; es decir, de acuerdo con los criterios utilizados no nos quedó claro si se trata de un marcador inferencial o de suposición. Consideramos como casos inferenciales aquellos en los que el hablante produce una información que es el resultado de una inferencia generada

11 La función retardataria, así como identificada en el análisis, podría coexistir con otras funciones de como que, sin embargo dada la escasez de casos y la presencia de interrupciones después del marcador no nos resultó posible formular hipótesis a este respecto. Resultan necesarios estudios adicionales que permitan arrojar luz sobre el tema.

${ }^{12}$ La clasificación de Fraser (1996) no incluye una categoría que explique esta función de como (que). 
por el contacto visual con algunos rastros de la acción (13), mientras que como casos de suposición aquellos en los que la información proviene de un proceso de razonamiento sobre algo que el hablante ya sabe (14).

(13) P2: Me sonó como arena a mí

P1: Sí / eso es como un muelle / ahí atráh

P2: Puede ser

(14) P2: O también podría ser que esah leñas son como que resultan como terminando de construir la casa

(15) P1: aa la ropa se la devuelve / aaa tienes razón / además tiene una sonrisa como contento / entre todas las cosas [que le pasaron]

El ejemplo (13) muestra el uso de como con función evidencial inferencial. Aquí, el marcador introduce dos elementos nominales arena y muelle que aparecen dibujados en la Figura 1a. En (14), en cambio, el hablante está especulando sobre la razón por la que hay varios troncos de madera en la Figura 1c. Esta especulación, en efecto, se basa en un proceso cognitivo distinto del caso mostrado en el ejemplo (13) ya que, aquí, no se trata de una inferencia concerniente a la naturaleza del objeto, sino que es un proceso que se basa en los conocimientos del hablante, quien toma en consideración las utilizaciones posibles que los personajes de las imágenes podrían haber hecho de los troncos de madera. Finalmente, en (15) no hemos conseguido identificar si se trata de una inferencia o de una suposición. La razón de esta duda depende del hecho de que el hablante a partir de un elemento visual (Figura 1b), la sonrisa, identifica un sentimiento del personaje de la tarea. La identificación de este sentimiento, sin embargo, de-

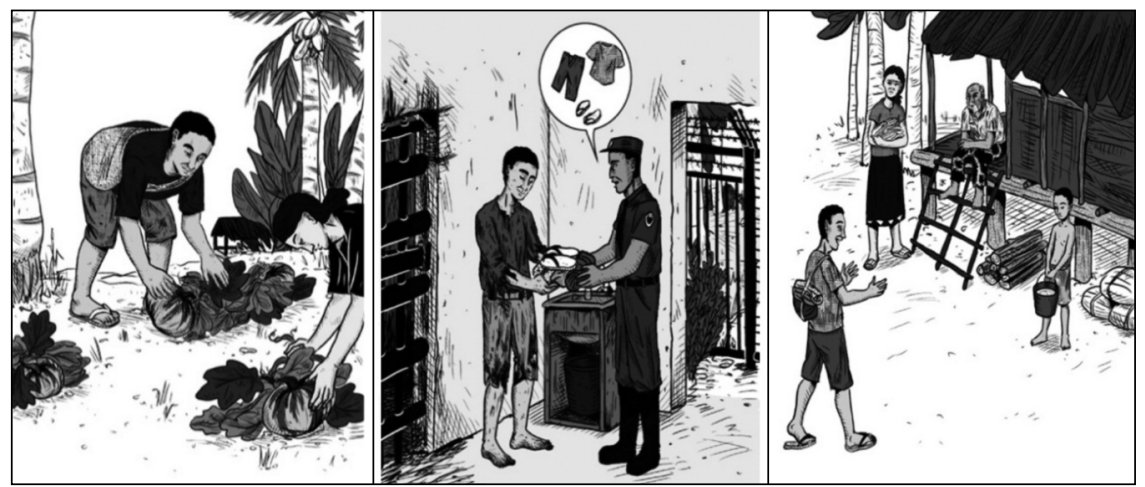


pende de los conocimientos previos del hablante con respecto a la historia que está construyendo y, por lo tanto, es también el resultado de un proceso de razonamiento.

Con respecto a la función de como (que) mencionada por Panussis Lyon y San Martín Núñez (2017), es decir, introductor de cita en discurso directo, en el presente estudio consideramos estos casos como pertenecientes a la función de suposición. Esta elección se debe al hecho de que en estos casos como (que) no se utiliza meramente para introducir un reporte literal, sino que señala lo que el hablante supone que un personaje de la tarea ha dicho a partir tanto de su conocimiento de situaciones reales similares a las dibujadas en las imágenes como de la historia construida hasta el momento de la enunciación (16). Por todo ello, el número de casos de esta función forma parte de los casos evidenciales de suposición.

(16) P2: [...] estaban tomando mucho / yyy uno de los tipo(s) - uno de los vecino(s) como que le dice aaa / al gallo que era temporero como oye vi a tu señora con otro gallo pero estaba como conversando con él $[\ldots]$

El último subdominio evidencial indirecto que hemos detectado es la evidencialidad de rumor. Discutiremos esta función a través de los ejemplos (17) y (18).

(17) P3: [...] yyy después de que lo llevan preso / eee / ya en la cárcel como que reflexiona de su actitud / y de la vida / y suh acciones / y después de salir / vuelve a familia pero la familia ya no lo acepta / y esa es la historia

(18) P2: [...] cuando está en la cárcel como que está como / mal / yyy / como extrañaba su familia / y reflexiona sobre la condena y todo esto / y después como que sale de la cárcel como que aprecia su libertad / y vuelve a su casa y no lo reciben tan bien / él pensaba que lo iban a recibir muy bien / pero después como que les cuenta que él estuvo en la cárcel y como que ha reflexionado sobre la vida / fin

El ejemplo (17) aparece durante el quinto y último paso de la tarea. El elemento que nos lleva a pensar en una posible función evidencial de rumor de como (que) depende de su correlación con el relato del mismo momento de la historia durante el cuarto paso de la tarea Family Problems Picture, mostrado en el ejemplo (18). En (17), P3 utiliza el mismo verbo utilizado por P2 en (18), reflexiona. Sin embargo, al usar como que, P3 quiere señalar una distancia con la información y manifiesta la voluntad de indicar que la acción descrita no es una interpretación propia. Esta distancia evidencial se refleja en cierta distancia epistémica, es decir, un compromiso reducido con respecto a la exactitud de la información, que el hablante crea al añadir como que. 


\subsection{Distribución de significados y funciones de como (que) en la muestra}

Después de una primera observación de los números (Cuadro 1), es evidente que la función evidencial indirecta de como (que) (que incluye, recordemos, las funciones de inferencia, suposición y de rumor) es claramente superior a todas las demás, ya que representa más del $50 \%$ del total de los casos.

En el corpus, el marcador como (que) aparece en 652 casos: 516 sin el complementador $(79,14 \%)$ y 136 con el complementador $(20,86 \%)$. Un primer análisis cuantitativo ha mostrado que el número total de apariciones de como (que) representa el 2,3\% del número total de palabras, es decir, 28 953. A modo de comparación, en el Corpus de Referencia del Español Actual (CREA), el porcentaje de aparición de como (que), 773465 formas, equivale solo al $0,5 \%$ del total de palabras del corpus (154212661 palabras $)^{13}$. Este resultado muestra la altísima frecuencia que tiene este marcador en el español oral de Chile. La comparación entre el porcentaje de apariciones entre ambos corpus puede apreciarse en el Cuadro 2:

CuAdro 2. Apariciones de como (que) en la muestra y en el Corpus de Referencia del Español Actual

\begin{tabular}{|c|c|c|c|c|c|}
\hline \multicolumn{3}{|c|}{ Muestra actual del español de Chile } & \multicolumn{3}{|c|}{ CREA } \\
\hline $\mathrm{N}^{\circ} \operatorname{como}(q u e)$ & $\mathrm{N}^{\circ}$ total de pp. & $\%$ & $\mathrm{~N}^{\circ}$ Como (que) & $\mathrm{N}^{\circ}$ total de pp. & $\%$ \\
\hline 652 & 28953 & 2,3 & 773465 & 154212661 & 0,5 \\
\hline
\end{tabular}

Del total de apariciones de como (que) en nuestro corpus, $428(65 \%)$ fueron emitidas por mujeres y $224(34,4 \%)$ por hombres. Del total de palabras emitidas por las mujeres (16521), los 428 como (que) equivalen a un 2,59\%. Por su parte, del total de palabras emitidas por los hombres (12171), los 224 como (que) equivalen a un 1,84\%. Esto muestra que, si bien la frecuencia de como (que) es alta para los dos grupos, son las mujeres quienes la usan con una frecuencia más destacada.

Sobre la frecuencia de aparición de como (que) en relación con la edad de los participantes, dado que la participación de estos fue desigual -del total de participantes, 12 (40\%) eran jóvenes (18-30 años), 14 (47\%) adultos (31-50 años) y solo 4 (13\%) mayores de 50 años- comparamos las formas emitidas por cada grupo etario en relación con el total de palabras

\footnotetext{
${ }^{13}$ Todos los datos estadísticos del CREA están tomados de: <http://corpus.rae.es/lfrecuen cias.html>.
} 
expresadas por ese mismo grupo. Así, en los jóvenes como (que) equivale al $3,62 \%$ de sus emisiones (428 de 11833 palabras); en los adultos al 1,43\% (183 de 12820 palabras); finalmente, en los mayores la expresión como (que) fue producida en un 0,95\% (41 de 4309 palabras). Desde un punto de vista descriptivo, entonces, parece ser que es el grupo etario joven el que utiliza como (que) de manera más frecuente y su uso desciende con la edad.

Un resultado interesante se produce al cruzar las variables de sexo y edad. Al hacerlo, podemos ver que las mujeres jóvenes utilizan como (que) en una proporción del 4,46\% (313 de 7018 palabras producidas por este grupo), las mujeres adultas en un 1,37\% (74 de 5401 palabras) y las mujeres mayores, en un $1 \%$ (41 de 4099 palabras). En el grupo de hablantes mujeres, en consecuencia, las jóvenes presentan una frecuencia de uso mucho mayor que en los otros grupos etarios (Gráfico 1). Entre los hombres, por su parte, los jóvenes utilizan como (que) en un 2,39\% (115 de 4812 palabras producidas por este grupo), los adultos en un 1,53\% (109 de 7124 palabras), mientras que los hombres mayores no expresaron ninguna de estas formas en sus 235 palabras emitidas. En este grupo por sexo, por lo tanto, son también los jóvenes quienes usan la forma en estudio con mayor frecuencia, aunque con menor diferencia que en el caso de las mujeres. Entre todos los grupos estudiados, las mujeres jóvenes son quienes utilizan como (que) con una frecuencia mayor, seguida de los hombres jóvenes, mientras que los hombres mayores son quienes menos la usan, seguidas de las mujeres mayores. Todos estos datos pueden apreciarse resumidamente en el Gráfico 1.

GRÁfICO 1. Uso de como (que) según sexo y edad (porcentajes en relación con el total de palabras emitidas por cada grupo)

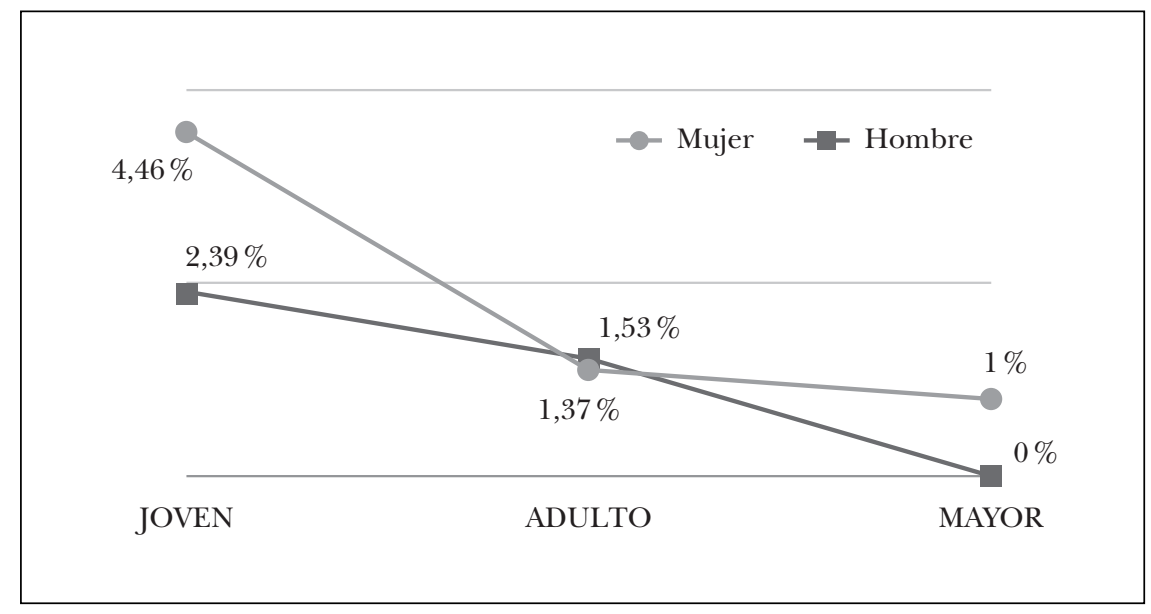


Para establecer si existen relaciones entre los grupos sociolingüísticos y las funciones expresadas por como (que) consideraremos solamente las funciones evidenciales (inferenciales, de suposición y de rumor), así como las funciones atenuadoras y aproximadoras. Dejaremos fuera, en consecuencia,

i) las funciones gramaticales (adverbio, conjunción y preposición) por no ser pertinentes para nuestros propósitos;

ii) la función retardataria, que no transmite un significado propiamente tal, sino que es un fenómeno propio de la actuación, y

iii) la función ejemplificadora, por presentar una cantidad muy escasa de apariciones en nuestro corpus.

No consideraremos tampoco para el análisis cuantitativo los 22 casos ambiguos entre las funciones evidenciales de inferencia y suposición. Para no extendernos en demasía, compararemos la producción de como (que) en estas funciones para los grupos conformados por sexo y edad [ (a) mujeres jóvenes, (b) mujeres adultas, (c) mujeres mayores, (d) hombres jóvenes, (e) hombres adultos, y (f) hombres mayores]. Finalmente, con el propósito de presentar datos que sean comparables, estableceremos las cantidades totales y, junto a ellas, su proporción en relación con el total de palabras producidas por el grupo sociolingüístico pertinente y, dado que en gran parte de los casos la cantidad de apariciones es muy escasa para expresarla en porcentajes, las cantidades proporcionales se expresan siguiendo la fórmula (como (que) / total de palabras del grupo) *1000. Estos resultados se indican en el Cuadro 3. Dado que el grupo conformado por los hombres mayores de 51 años no produjo casos de como (que), no se presentan en el cuadro.

Lo primero que resalta de la observación de estos datos es que la función evidencial de suposición constituye el significado que más frecuentemente es transmitido por como (que) para todos los grupos sociolingüísticos definidos, mientras que el significado evidencial referido es la función menos expresada igualmente en todos los grupos que conforman la muestra. Dentro de las funciones no evidenciales, los resultados para la atenuación y la aproximación son muy similares para todos los grupos sociolingüísticos, siendo los usos aproximativos ligeramente superiores en ambos grupos de jóvenes, y con mayor diferencia en los hombres adultos y mujeres mayores. Tanto en los usos evidenciales como en el total de expresiones de como (que) en las funciones consignadas de la muestra, son las mujeres jóvenes quienes manifiestan un mayor uso absoluto y proporcional al total de palabras del grupo, mientras que los hombres mayores, como ya se ha dicho, son el grupo sociolingüístico que menos hace uso de este marcador, seguidos por las mujeres mayores, lo que es indicador de que -al menos en la muestra recopilada- la edad parece ser un rasgo importante en la variación. 
CuAdro 3. Usos de como (que) según función por cada grupo sociolingüístico

\begin{tabular}{|c|c|c|c|c|c|}
\hline Función & $\begin{array}{c}\text { Mujeres } \\
\text { jóvenes } \\
\text { (7 } 018 \text { pp.) }\end{array}$ & $\begin{array}{c}\text { Hombres } \\
\text { jóvenes } \\
\text { (4812 pp.) }\end{array}$ & $\begin{array}{c}\text { Mujeres } \\
\text { adultas } \\
\text { (5 401 pp.) }\end{array}$ & $\begin{array}{l}\text { Hombres } \\
\text { adultos } \\
\text { (7 124 pp.) }\end{array}$ & $\begin{array}{c}\text { Mujeres } \\
\text { mayores } \\
\text { (4099 pp.) }\end{array}$ \\
\hline Inferencial & $42(5,99)$ & $15(3,11)$ & $8(1,48)$ & $17(2,38)$ & $8(1,95)$ \\
\hline Suposición & $141(20,11)$ & $32(6,64)$ & $38(7,03)$ & $35(4,9)$ & $9(2,19)$ \\
\hline Rumor & $11(1,57)$ & $1(0,21)$ & 0 & $1(0,14)$ & 0 \\
\hline Evidencial Tot. & $194(27,64)$ & $48(9,97)$ & $46(8,52)$ & $53(7,44)$ & $17(4,15)$ \\
\hline Atenuador & $33(4,71)$ & $17(?$ & $6(1$ & $6(0$ & $2(0,49)$ \\
\hline Aproximador & $38(5,42)$ & $20(4,15)$ & $5(0,92)$ & $14(1,96)$ & $11(2,68)$ \\
\hline Total & $265(37,76)$ & $85(17,66)$ & $57(10,56)$ & $73(10,25)$ & $30(7,32)$ \\
\hline
\end{tabular}

Dentro de los usos evidenciales específicos, para la función evidencial inferencial las mujeres jóvenes son el grupo que más expresa este significado, seguido por el grupo de los hombres jóvenes. Los hombres mayores (como en todos los casos) son el grupo que menos expresa esta función seguidos por las mujeres adultas. Para la función evidencial de suposición, nuevamente las mujeres jóvenes son quienes más la manifiestan, seguidas en este caso por las mujeres adultas; hombres mayores y hombres adultos son quienes menos la expresan. Finalmente, en el caso de la evidencialidad referida, solo tres grupos la manifestaron, resaltando una vez más las mujeres jóvenes como el grupo con el mayor número de casos utilizados. Dentro de las funciones no evidenciales, con respecto a la función atenuadora, son las mujeres jóvenes quienes la expresan con más frecuencia, seguidas de los hombres jóvenes; los grupos de mayores, tanto hombres como mujeres, apenas presentan casos en conjunto. En relación con la función aproximadora, finalmente, la situación es muy similar y esta función es expresada fundamentalmente por mujeres y hombres jóvenes.

Pasaremos ahora al análisis de las funciones pragmáticas observadas durante la tarea y sus diferentes pasos. Para hacer esto, nuevamente consideraremos solo las funciones evidenciales (inferenciales, de suposición y de rumor), así como las funciones atenuadoras y aproximadoras y, como en los puntos anteriores, dejaremos fuera los casos de ambigüedad entre significados de inferencia y de suposición.

En una aproximación general, se puede establecer que, tal como muestra el Gráfico 2, la función evidencial de suposición es el significado más transmitido por como (que) en el conjunto de la tarea, con un total de 255 apariciones $(50 \%)$. Le siguen la función evidencial inferencial (90 casos, 
$17,6 \%)$, la función aproximadora (88 casos, 17,3\%), la función atenuadora (64 casos, 12,5\%) y, finalmente, la función evidencial referida (13 casos, $2,5 \%$ ). Las funciones evidenciales en conjunto suman 358 casos y conforman el 70,1\% del total de casos de como (que).

GRÁFICO 2. Funciones pragmáticas totales en la tarea

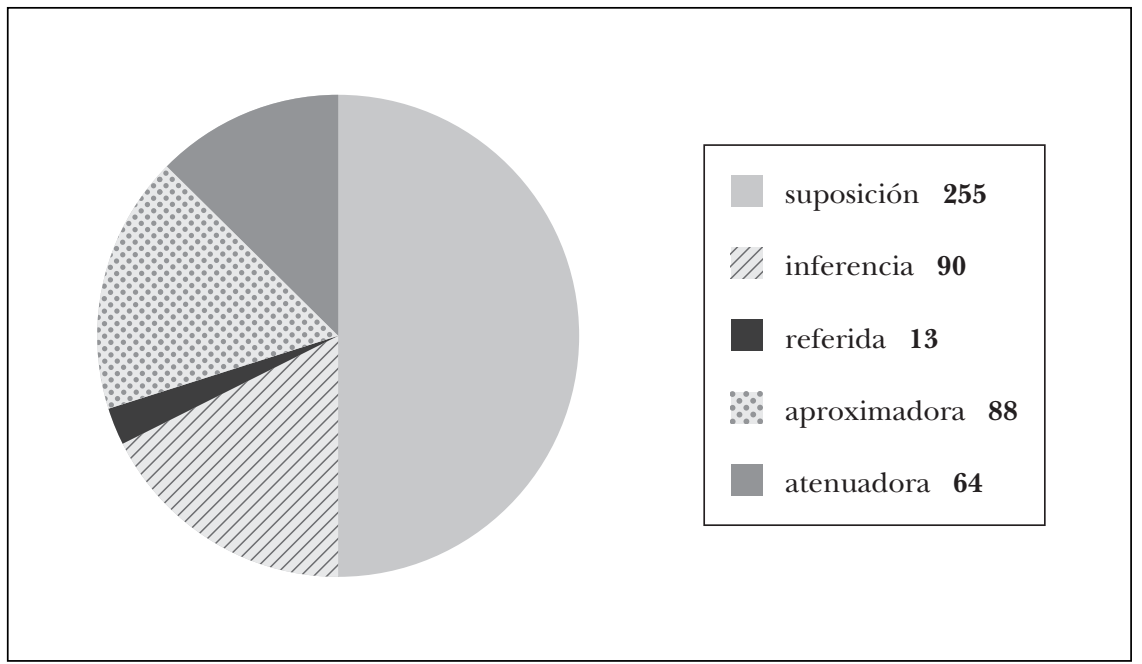

Sobre los pasos de la tarea en que cada una de las funciones se expresa con mayor frecuencia, los datos resumidos pueden encontrarse en el Cuadro 4, a continuación. Recordemos que el paso 1 consistía en la descripción de 5 imágenes escogidas al azar de un universo de 16 posibles; el paso 2 implicaba la construcción conjunta de una historia a partir de las imágenes; el paso 3 consistía en la narración en primera persona de la historia al trabajador de campo por parte de uno de los participantes; en el paso 4 uno de estos participantes originales contaba la historia en tercera persona a un hablante que no participó en las primeras tres etapas; en el quinto paso, finalmente, el hablante que acaba de enterarse de la historia se la cuenta al trabajador de campo. El Cuadro 4 presenta las frecuencias absolutas y los porcentajes de la funciones pragmáticas analizadas en relación con los pasos de la tarea Family Problems Picture.

Como se observa en el cuadro, la mayor cantidad de expresiones de como (que) con los valores pragmáticos especificados se dan en el paso 1 de la tarea, mientras que la menor cantidad se encuentra en el paso 3. La función evidencial de suposición se centra fundamentalmente en los pasos 1 y 2 y está prácticamente ausente en los pasos 3 y 5 . La función evidencial 
CuAdro 4. Usos de como (que) según función por cada paso de la tarea

\begin{tabular}{lcclccr} 
& Paso 1 & Paso 2 & Paso 3 & Paso 4 & Paso 5 & Total \\
\hline Suposición & $142(55,7 \%)$ & $76(29,8 \%)$ & $5(2 \%)$ & $31(12,2 \%)$ & $1(0,4 \%)$ & $\mathbf{2 5 5}$ \\
Inferencia & $67(74,4 \%)$ & $15(16,7 \%)$ & $2(2,2 \%)$ & $6(6,7 \%)$ & 0 & $\mathbf{9 0}$ \\
Referida & 0 & 0 & 0 & $3(23,1 \%)$ & $10(76,9 \%)$ & $\mathbf{1 3}$ \\
Aproximador & $51(58 \%)$ & $10(11,4 \%)$ & $4(4,5 \%)$ & $15(17 \%)$ & $8(9,1 \%)$ & $\mathbf{8 8}$ \\
Atenuador & $39(60,9 \%)$ & $17(26,6 \%)$ & 0 & $6(9,4 \%)$ & $2(3,1 \%)$ & $\mathbf{6 4}$ \\
\hline Total & $\mathbf{2 9 9 ( 5 8 , 6 \% )}$ & $\mathbf{1 1 8 ( 2 3 , 1 \% )}$ & $\mathbf{9 ( 1 , 8 \% )}$ & $\mathbf{6 1 ( 1 2 \% )}$ & $\mathbf{2 1 ( 4 , \mathbf { 1 } \% )}$ & $\mathbf{5 1 0}$ \\
\hline
\end{tabular}

de inferencia se da también con mayor frecuencia en el paso 1 de la tarea, seguida ahora lejanamente por sus apariciones en el paso 2, estando nuevamente casi ausente en su totalidad en los pasos 3 y 5 . Que la suposición y la inferencia se expresen mayoritariamente en el primer paso es coherente con la naturaleza de este paso de la tarea, en que los hablantes se enfrentan a imágenes de personas cuya identidad y acciones les son desconocidas. Que la inferencia baje en el segundo paso, pero la suposición se mantenga con alta frecuencia en este mismo se explica porque los hablantes ya no se centran en tratar de entender las imágenes sino en construir una historia coherente, para lo que se basan en sus conocimientos previos. En contraposición a esto, la función evidencial de rumor se da exclusivamente en los pasos 4 y 5 de la tarea. Esto es también esperable, ya que la tarea se trata aquí de relatar a otro una historia. Las funciones pragmáticas no evidenciales de aproximación y atenuación se dan también con mayor frecuencia en el paso 1 y se encuentran casi totalmente ausentes en el tercero.

Si miramos finalmente la cantidad de manifestaciones de cada función por paso, veremos que en todos ellos predomina la expresión de la función evidencial de suposición, con la sola excepción del paso quinto, en que predomina la función evidencial referida y la primera está casi completamente ausente.

\section{Conclusiones}

Como ya mencionamos, los usos pragmáticos del marcador como (que) están documentados tanto en las variedades americanas del español (Kany 1945: 383; Montes 1980; Jørgensen 2012; Puga 1997, 2014; Panussis Lyon 
y San Martín Núñez 2017) como en las variedades peninsulares (Jørgensen 2012). A pesar de la amplia difusión de como (que) en las variedades de habla hispana, los resultados del análisis cuantitativo (cf. § 2.3) sugieren que el español de Chile podría presentar un uso más extenso de este marcador con respecto a las otras variedades del español, aunque afirmar esto requeriría un estudio comparativo de su frecuencia en relación con los otros dialectos.

Desde el punto de vista de su distribución sociolingüística, parece especialmente relevante que la expresión como (que), independientemente de su significado, se exprese de manera tan frecuente entre los hablantes jóvenes, sobre todo por las mujeres de este grupo etario. Que el uso de este marcador sea casi en todos los casos más marcado en las mujeres y que su uso vaya disminuyendo con la edad de los hablantes nos parece también algo relevante. Una pregunta interesante que se puede plantear es si esta distribución corresponde a un patrón sociolingüístico estable o si, por el contrario, da muestras de un proceso de cambio lingüístico, cuestión que debemos dejar planteada para futuras investigaciones que establezcan comparaciones diacrónicas. Por último, ponemos de relieve que en nuestro corpus sea la función evidencial de suposición la que mayormente es transmitida por como (que) y, nuevamente, de manera especialmente destacada en el grupo de mujeres jóvenes.

Otras consideraciones conclusivas están relacionadas con las funciones evidenciales de como (que). En primer lugar, cabe especificar que el número relevante de usos de como (que) con función evidencial indirecta presente en los datos no debe llevar a conclusiones precipitadas en cuanto a los usos predominantes de este marcador en la variedad objeto de estudio. Consideramos, en efecto, que este número puede depender estrictamente del tipo de tarea utilizada para elicitar los datos, es decir, una tarea que fue diseñada para estimular la evidencialidad y el reporte de habla (cf. §2.1). En segundo lugar, si por un lado creemos que usos evidenciales directos (visual y sensorial) de como (que) son improbables, por el otro, consideramos posible que otros estudios identifiquen funciones evidenciales referidas de 'tercera mano' y 'folclore' ${ }^{14}$ (Willett 1988), que en este trabajo, debido a la naturaleza de la tarea utilizada, no han sido identificadas.

Finalmente, hasta donde tenemos conocimiento, no se han descrito usos evidenciales de como (que) en otras variedades del español hasta la fecha. Esta última consideración no pretende indicar la ausencia de tales usos en otras variedades de español, sino que hace hincapié en la necesidad de realizar más estudios en esta dirección.

${ }^{14}$ En los términos de Willett (1988), la definición «evidencialidad de rumor», que se adoptó en este artículo por razones analíticas, es «evidencialidad referida de segunda mano». 


\section{BIBLIOGRAFÍA}

ACín Villa, Esperanza (1987): «Construcciones con “como” en español moderno», RILCE 3/1, 25-59.

Ajjmer, Karin y Anne-Marie Simon-Vandenbergen (2006): Pragmatic Markers in Contrast, Ámsterdam/Londres: Elsevier.

Aikhenvald, Alexandra (2004): Evidentiality, Oxford: Oxford University Press.

BRINTON, Laurel J. (1996): Pragmatic Markers in English: Grammaticalization and Discourse Functions, Berlín: Mouton de Gruyter.

CERVERA Rodríguez, Ángel (2009): «Tratamiento gramatical y comportamiento discursivo de como en el español actual», Español Actual 91, 57-98.

CHAFE, Wallace y Johanna NicHOLS (eds.) (1986): Evidentiality: The Linguistic Coding of Epistemology, Norwood: Ablex.

Channell, Joanna (1994): Vague Language, Oxford: Oxford University Press.

Cutting, Joan (ed.) (2007): Vague Language Explored, Nueva York: Palgrave MacMillan.

FrASER, Bruce (1996): «Pragmatic Markers», Pragmatics 6/2, 167-190.

- (2006): «Towards a Theory of Discourse Markers». En Kerstin Fischer (ed.), Approaches to Discourse Particles, Ámsterdam: Elsevier, 189-204.

Hernando CuAdrado, Luis Alberto (2002): «Sobre el funcionamiento de como en español», Revista de Filología Románica 19, 325-340.

Hopper, Paul J. y Elizabeth TraugotT (2003): Grammaticalization. Cambridge Textbooks in Linguistics, Cambridge: Cambridge University Press.

IgLesias Bango, Manuel (2006): «Una vez más, Bello: Como y los marcadores del discurso». En Antonio Roldán Pérez (coord.), Caminos actuales de la historiografía lingüistica. Actas del V Congreso Internacional de la Sociedad Española de Historiografia lingüistica, Murcia: Universidad de Murcia, vol. 2, 815-832.

JøRGENSEN, Annette Mire (2012): «Funciones del marcador pragmático como en el lenguaje juvenil español y chileno». En María Elena Placencia y Carmen García (eds.), Pragmática y comunicación intercultural en el mundo hispanohablante, Londres: Rodopi, 209-230.

- y Anna-Brita STENSTRøM (2009): «Dos marcadores pragmáticos contrastados en el lenguaje juvenil: El inglés like y el español como», Español Actual 92, 103-121.

KANY, Charles (1945): American-Spanish Syntax, Chicago: University of Chicago Press.

Montes, José Joaquín (1980-1981): «Sobre el como de atenuación», Boletín de Filología XXXI, 667-675.

NuYTS, Jan (2001): Epistemic Modality, Language and Conceptualization. A Cognitivepragmatic Perspective, Ámsterdam: John Benjamins.

Panussis Lyon, Constanza y Abelardo SAn Martín Núñez (2017): «Como (que) y sus funciones discursivas en el habla santiaguina: análisis pragmático y sociolingüístico», Revista de Lingüistica Teórica y Aplicada 55/2, 39-61. 
Plungian, Vladimir (2001): «The Place of Evidentiality within the Universal Grammatical Space», Journal of Pragmatics 33, 349-357.

PugA, Juana (1997): La atenuación en el castellano de Chile: Un enfoque pragmalinguístico, Valencia: Estudios Iberoamericanos.

San Roque, Lila, Lawren Gawne, Darja Hoenigman, Julia Colleen Miller, Alan Rumsey, Stef Spronck, Alice Carroll y Nicholas Evans (2012): «Getting the Story Straight: Language Fieldwork Using a Narrative Problem-Solving Task», Language Documentation \& Conservation 6, 135-174.

WiLlet, Thomas (1988): «A Cross-linguistic Survey of the Grammaticalization of Evidentiality», Studies in Language 12, 51-97. 